\title{
De reactivo biológico al animal sintiente: el bienestar animal como cambio de paradigma en la investigación biomédica y su impacto en los resultados
}

\section{From biological reagent to sentient animal: animal welfare as a paradigm shift in biomedical research and its impact on results}

\author{
Maschi Fabricio Alejandro ${ }^{1}{ }^{1 *}$, Carbone Cecilia $^{1}$, Ferrari Héctor Ricardo ${ }^{2}$
}

1. Laboratorio de Animales de Experimentación (LAE), Facultad de Ciencias Veterinarias. 2. Cátedra de Etología. Facultad de Ciencias Naturales y Museo. Universidad Nacional de La Plata. Argentina.

* Correo electrónico del autor de contacto: fmaschi@fcv.unlp.edu.ar

\begin{abstract}
Resumen
El uso de animales como reactivos biológicos para investigación científica se ha justificado desde los inicios de la civilización, cuando solo servían para satisfacer las necesidades del hombre. El concepto de reactivo biológico ha cambiado al cobrar relevancia el bienestar animal, una problemática específica de la relación humano-animal referida inicialmente a los animales de producción y que ha impactado en todos los usuarios de animales. Se logró avanzar en el conocimiento de las especies, sus necesidades y en la comprensión de su comportamiento. Con esta redefinición del animal surge un nuevo objeto de estudio para la ciencia: el estado del animal en sus intentos de lidiar con su entorno y de afrontar las demandas del ambiente. Como consecuencia, se han realizado enriquecimientos del ambiente sin suficientes estudios previos llevando a la obtención de resultados erróneos. Por lo tanto, es imperativo trabajar con indicadores específicos de bienestar que permitan diagnosticar y remediar las condiciones de pobre bienestar. Los animales provistos a los investigadores deberían contar con certificaciones sobre la cepa, condiciones de crianza y, además, tipo y modalidad de enriquecimiento e indicadores de bienestar animal antes de iniciar su utilización. En el futuro, el bienestar animal no solo debe estar en la nota ética, sino que debe ser parte inexcusable de la sección metodológica de los estudios experimentales.
\end{abstract}

\section{Palabras clave}

Animales de experimentación, bienestar animal, enriquecimiento ambiental, sintiencia

\begin{abstract}
The use of animals for scientific research has been justified since the beginnings of civilization, when they were considered as a biological reagent and only served to meet the needs of humans. This concept changed when ethical considerations towards their use as experimental beings began to have greater relevance. The birth of animal welfare has impacted the entire scientific community and all animal users, allowing a greater understanding of the needs and behavior of different species. From this redefinition of the animal as a biological reagent a new object of study arises: the relationship of the animal with its environment and its attempts to cope with the demands of the environment. Several attempts have been made to enrich the environment of experimental animals in order to improve their welfare but often in a poorly studied manner, and this has produced erroneous scientific results. Therefore, it is imperative to work with specific indicators of well-being that diagnose and remedy poor welfare conditions, and accurately communicate the conditions of the groups from which the statements are made. Thus, animals provided to researchers should be accompanied with certifications about strain, breeding conditions, type of enrichment and animal welfare indicators, prior to their use. In the future, animal welfare should not only be on the ethical note of experimental studies, but also be an indisputable part in the methodological section.
\end{abstract}

\section{Key words}

Animal welfare, environmental enrichment, experimental animals, sentience

Fecha de recepción: 28/11/2018

Fecha de revisión: 04/02/2019

Fecha de aprobación: 20/05/2019
ANALECTA VeT 2019; Enero-Junio; 39(1):21-31

Impresa ISSN 03655 14-8 Electrónica ISSN 1514-2590

doi.org/10.24215/15142590eo34 


\section{Introducción}

El uso de animales para investigación, en especial los criados en bioterios que se destinan para esa finalidad exclusivamente, forma parte de un entramado ideológico que se estructura en torno a la noción de que aquellos son un medio para un fin. Idealmente, ese fin es un bien para otros seres, los humanos fundamentalmente. Implica una serie de resignificaciones que se equilibran en la tensión entre considerarlo similar al humano (para que los resultados sean útiles) y diferentes del humano (para que su uso no genere contradicciones éticas y morales) (De Mello, 2012).

El uso de animales en investigaciones es una decisión ética que tiene que ver con las nociones del bien y del mal y, como tal, escapa a lo que pueda construir el quehacer científico en cuanto a afirmaciones. Así, muchos de los lineamientos propuestos por las organizaciones científicas invitan a preguntarse: ctiene sentido este uso que doy a los animales? (ASAB, 1986). Históricamente, esa decisión ética se construyó con un punto de inicio, difusamente reconocible, en la época en que los animales eran considerados máquinas; actitud que, desde un principio, encontró opositores (De Mello, 2012).

No es un tema reciente que la sociedad se plantee la cuestión ética en el uso de los animales para investigación. Desde un principio no se contó con la existencia de un criterio hegemónico. Entre 1903 y 1910, a raíz de una vivisección realizada en la Universidad de Londres por William Bayliss del Departamento de Fisiología, un grupo de proteccionistas contra el uso de animales iniciaron un movimiento que pasó a la historia como, entre otros nombres, "los motines del perro marrón". Tuvieron su momento de mayor exposición cuando el 10 de diciembre de 1907, manifestantes a favor del uso de animales, se enfrentaron en el centro de Londres con sufragistas, policías y sindicalistas, provocando disturbios y peleas callejeras extendidas (Mason, 1997).

Como todo proceso histórico, cada etapa es consecuencia de lo sucedido anteriormente. En cierta forma, los resultados científicos se vuelven obsoletos apenas publicados y sientan las bases para desarrollos que, partiendo de ellos, los trasciendan.

Lo mismo ha ocurrido con los animales de experimentación; las propias investigaciones en los que estos fueron utilizados acabaron por transformar nuestra concepción a una imagen diferente del animal máquina. Paradójicamente, a modo de las demostraciones por el absurdo de las matemáticas, considerar a los seres vivos como reactivos biológicos, es parte de lo que nos lleva a negar que sean exclusivamente reactivos. Parte de esa negación, produce en el campo científico el advenimiento de la ciencia del bienestar animal (Cardoso \& Almeida, 2014; Maschi, 2017).

\section{El bienestar animal: generalidades y componentes}

Esta temática, relativamente nueva, ha impactado de una u otra manera en todos los usuarios de animales para investigación de la comunidad científica, ya sea productores o investigadores que los mantengan bajo experiencia. Esto ha influido directa o indirectamente en las investigaciones porque se ha avanzado mucho en el conocimiento de las especies y sus necesidades. Parte de ese avance, es entender que un animal no es solo su anatomía y su fisiología sino también, y especialmente, su comportamiento. Es a partir de esta redefinición del animal que surge un nuevo objeto de estudio para la ciencia: la relación del animal con su entorno y, a su vez, una nueva dimensión en la que se cuestiona no solo lo que se hace con él, sino también cómo está y siente en relación con lo que se le hace.

Es decir, como está el animal en relación con sus intentos de afrontar las demandas del ambiente y lidiar con él. Estos conceptos componen lo que se denomina bienestar animal (Broom, 2016).

Esta situación dispara una serie de interrogantes: ¿todos comprendemos las necesidades de cada especie en particular?, y si nos referimos a los más utilizados como son los roedores, ¿todas las cepas de ratas o ratones se comportan igual?, ¿tienen todas las mismas necesidades?, ¿se puede estandarizar una forma de manejo para la mejora en su bienestar?, ¿cómo sabemos que hemos logrado esa mejora?, des correcto preocuparse solamente por el bienestar de los animales en producción y no por los que están bajo experimentación? y, por último, lo que adquiere relevancia en este contexto ciafecta los resultados experimentales? (Maschi, 2017).

¿Por qué preocuparse por el bienestar?; si no tenemos ninguna obligación moral o ética hacia los animales para brindarles bienestar, no hay razón entonces para considerar si este es bueno o malo. Por otro lado, si tenemos obligaciones morales, entonces debemos ser capaces de evaluar el bienestar animal cuando trabajamos con ellos (Singer, 2015).

Aceptamos aquí que tenemos esas obligaciones y daremos un paso más. Al procurar bienestar a los animales de investigación, como en verdad estamos teniéndolos en cuenta como lo que son y no con una visión simplificada (el reactivo, la máquina), mejoraremos nuestros resultados.

El bienestar animal tiene dos componentes: físico y del comportamiento. El primero se manifiesta por un estado excelente de salud y el segundo se manifiesta por el desarrollo comportamental completo, específico de especie o cepa dada, junto con la ausencia de comportamientos atípicos para la especie. El bienestar del comportamiento refleja el bienestar psicológico, de manera 
que estos dos términos llegan a ser sinónimos para nuestro uso. Para crear un estado de bienestar, cada animal necesita, entre otras cosas, un ambiente social en el cual puede gozar de un mínimo de contactos básicos y de relaciones sociales positivas. El comportamiento social permite a los animales adaptarse a las condiciones de alojamiento. Enjaular a los animales, solos, por pareja o por grupos, debería ser hecho de manera de crear un ambiente estimulante apropiado para cada especie (Hughes, 1976).

El término bienestar se confunde o aplica como sinónimo de "estar bien” y viceversa, lo cual no es correcto. El "estar bien" se relaciona con la calidad de vida del animal en el corto plazo, mientras que el bienestar está referido al largo plazo. Como ejemplo, para mejorar el bienestar de un animal, puede ser necesario reducir temporalmente su "estar bien" con la práctica de una vacunación. Como resultado de la práctica, el bienestar aumentará debido a la reducción del riesgo para el animal de padecer una enfermedad, resultando así en una vida larga y de mejor calidad (vida digna). El "estar bien" del animal al igual que el bienestar deben ser siempre evaluados por la persona responsable de su cuidado (De Grazia, 1996).

La Organización Mundial de la Salud define salud como el "estado de completo bienestar de un organismo vivo, tanto desde el punto de vista mental como físico y social", y no solo se refiere a la ausencia de enfermedad (OMS). Sin embargo, la mayoría de los científicos se refiere a la salud animal como una condición inferior al bienestar ya que, por ejemplo, los animales pueden estar perfectamente saludables pero aun así tener una pobre calidad de vida si se encuentran alojados en ambientes incorrectos (Baumans, 2007).

Broom (1986) describe el bienestar animal como "el estado en el cual se encuentra un animal que trata de adaptarse a su ambiente". De esta definición se desprende que el bienestar de un animal es seriamente afectado por una falla de adaptación, a pesar de cuanto haya hecho ese individuo para lograrlo y qué tan bien o mal le haya ido en ese proceso. Entre sus considerandos, Broom destaca que: a) el bienestar es una característica de un animal y no algo que se le otorga; b) puede variar desde muy pobre a muy bueno; c) debería poder medirse de una manera científica, independientemente de las consideraciones éticas; d) las medidas de cuán difícil le ha resultado a un individuo adaptarse dan una idea de cuán pobre es su bienestar; e) el conocimiento de las preferencias de un animal otorga una información sumamente importante para lograr que tenga un buen bienestar, por lo tanto las mediciones directas de cómo se encuentra se deben utilizar para mejorar el mismo.

Como los animales emplean muchas estrategias para lograr adaptarse al medio, cuando fallan en ese proceso, se producen consecuencias que pueden ser medidas y que van a indicar que el bienestar es pobre. Por ejemplo, el hecho de que solo un parámetro como el crecimiento sea normal, no significa que el bienestar sea bueno; es por eso que al existir variedades de estrategias y resultados en el proceso de adaptación, deben medirse más de un tipo de indicadores (Broom, 1986).

La Comisión Brambell Rogers (1965) además emitió un informe en el que se describieron las "cinco libertades" de los animales domésticos y que representan la capacidad de los mismos de "darse vuelta, asearse, levantarse, acostarse y estirarse", fácilmente.

Más tarde, la World Veterinary Association (1989) adoptó sus propias cinco libertades, que se aplican a todas las especies y que están basadas en las del Britain's Farm Animal Welfare Council (FAWC). Estas definen los estados ideales para los animales e incluyen ser libres: 1) de hambre, sed y malnutrición; 2) de incomodidad física y dolor; 3) de sufrimiento, heridas y enfermedades; 4) de miedo y angustia prolongados; 5) para expresar un comportamiento normal (Turner, 1999).

Existen otros marcos de referencia cuando se habla de bienestar animal; sin embargo, aquí se hará hincapié en "las cinco libertades" por ser el concepto más difundido y porque no es antagonista de los otros desarrollos, que son más bien propuestas que extienden y superan estos lineamientos. En cierta forma, las "cinco libertades" aparecen como el mínimo aceptable (Mellor, 2016).

Este marco general, entendido como un conjunto de cinco grandes lineamientos, es aplicable a todos los animales mantenidos en cautiverio. Las primeras cuatro libertades se refieren a experiencias aversivas. De todos modos, es posible que un animal esté enfermo sin que él lo perciba, aunque podrían estar afectadas sus funciones naturales. Por ejemplo, si una rata preñada se infecta con el virus Kilham, la infección no la afectará directamente, aunque si atraviesa la barrera placentaria, pueden producirse abortos o malformaciones en los fetos (Baumans, 2007).

Asimismo, se debe tener en cuenta que si un animal está sufriendo, lo más probable es que no pueda sobreponerse a una situación difícil (Hawkins, 2002). Con buen alojamiento y buenas prácticas de manejo se minimizan las condiciones que llevan al miedo y angustia entre los individuos. En ambos casos las funciones naturales del animal están comprometidas.

Si bien sería un tema de largo debate cuestionar qué es el "comportamiento normal", (Segerdahl, 2007), los ambientes estériles en los bioterios contribuyen a expresiones de "comportamientos anormales" en la mayoría de las especies; en cambio, los ambientes que simulan condiciones naturales y/o promueven la expresión de comportamientos típicos de la especie, siempre serán beneficiosos (Hau \& Shapiro, 2007; ISAE, 2017). 
Se considera que las normas establecidas desde hace 50 años cumplen con los requerimientos de alojamiento óptimo para los animales de experimentación. Muchas de las mejoras en las condiciones de mantenimiento y las normas de manejo fueron establecidas, primariamente, para reducir las variables y optimizar la reproducibilidad de los resultados experimentales (Lang \& Vessell, 1976). Durante la última década, los esfuerzos fueron dirigidos a considerar las necesidades sociales y de comportamiento de los animales mantenidos en el laboratorio (Baumans, 2007).

Años atrás, se aceptaba que conejos y roedores de laboratorio tenían relativamente pocos requerimientos, con excepción de las necesidades esenciales de alojamiento, manejo y nutrición. Se hizo énfasis, entonces, sobre el control del ambiente, dejando de lado los otros aspectos. Algunos criterios que se han usado para evaluar objetivamente el bienestar son la ganancia de peso, el comportamiento general y el peso de las glándulas adrenales (Chamove, 1989).

\section{Valoración del estado de bienestar}

Habiendo definido el concepto de bienestar y su problemática, es necesario evaluarlo y, según Sorensen (2007), la mejor forma de hacerlo es siguiendo el método científico. La interpretación de mediciones realizadas va a depender de dos tipos de enfoques: científico y filosófico.

Según el enfoque científico, si los valores básicos obtenidos son objetivos, como lo es el buen funcionamiento biológico o la posibilidad de que los animales realicen comportamientos naturales, esto puede ser medido a través de parámetros reproductivos, prevalencia de enfermedades, medición de niveles de cortisol y observación de comportamientos estereotipados. Según el enfoque filosófico, los valores básicos obtenidos son de naturaleza subjetiva y están relacionados con el estado natural interno del animal, tales como sentimientos y preferencias. Solamente será posible evaluarlos a través de ciertos comportamientos del animal, que nos darán una medida indirecta. En la última década, se han desarrollado métodos basados en las expresiones faciales y el desvío cognitivo relacionado con los sentimientos, que permiten inferir algunos estados internos (Langford et al., 2010; Mendl et al., 2009).

La evaluación del estado de bienestar en los animales se realiza a través de los llamados indicadores de bienestar, empleando para ello el monitoreo de comportamientos apropiados y parámetros fisiológicos. De esta manera se puede decir que cada especie o cepa en cuestión tiene "un apropiado bienestar". Ese nivel hipotético de bienestar ideal podría definirse como el estado de los animales cuando se alcanzan las necesidades nutricionales, de salud, medioambientales, comportamentales y mentales (Mellor \& Reid, 1994).
Los tres componentes principales de un estado ideal de bienestar son el estado físico, el psicológico y el fisiológico/bioquímico. El estado físico se caracteriza por un buen nivel de aptitud física, sin incapacidades que puedan producir incomodidad o miedo o que tengan un impacto en dicha condición física y que produzcan temor. Los indicadores observables de la condición física de un animal son, entre otros: la postura del cuerpo, el pelaje, el peso y la presencia y severidad de cojeras. El estado psicológico se caracteriza porque el animal despliega un rango apropiado de comportamientos de acuerdo con lo que es conocido para esa cepa o especie. Los indicadores de que este estado se está deteriorando son, entre otros, el aumento de la agresión hacia sus compañeros de habitáculo, huidas o retiradas, desarrollo de estereotipias y ciertos cambios en el uso del enriquecimiento. El estado fisiológico se caracteriza porque los niveles de estrés o distrés no sobrepasan a los que podrían ocurrir en el curso de interacciones sociales normales. Los indicadores serían parámetros fisiológicos como frecuencia cardíaca, frecuencia respiratoria y niveles de hormonas como los corticosteroides los que no siempre indicarían estrés significativo (Fraser, 2004).

Una buena elección de indicadores de bienestar debe incluir la combinación de los tres estados mencionados, para permitir una correcta evaluación de interpretación del estado de un animal (Hawkins et al., 2011).

Dawkins (1990) propone que el bienestar involucra los sentimientos subjetivos de los animales y Duncan (1998) sostiene que el bienestar está gobernado por los sentimientos, los cuales deberían medirse para asegurar el bienestar animal.

A lo que hacen referencia estos y otros autores es a la sentience, traducido al español como "sintiencia", sensación, sentimiento más allá de la percepción mental (Chandrasekera, 2016).

El reconocimiento de la existencia de los sentimientos en animales es entendido como la capacidad de experimentar emociones tales como alegría, placer, dolor y miedo. La capacidad de sentir estados positivos y negativos es lo que impulsa el movimiento cada vez más importante del bienestar y justifica la existencia de leyes de protección animal (Proctor, 2012).

Los sentimientos en animales son difíciles de medir, pero si se asume que un animal prefiere vivir situaciones placenteras y evitar las negativas o desagradables, se pueden estimar los sentimientos midiendo las preferencias del animal (Sorensen, 2007).

Estos estados afectivos se entienden como una adaptación, orientada a evitar castigos y obtener recompensas. Una de las formas en que las emociones contribuyen al bienestar, y de hecho a la organización del comportamiento, es priorizando acciones. Las conductas que no pueden compararse por sus mecanismos o resultados (como comer, refugiarse, buscar conespecíficos, etc.), sí pueden serlo por las emociones o estados 
de ánimo asociados con ellas. Entonces, un comportamiento relacionado con lo social y otro relacionado con lo alimentario, pueden compararse sobre la base del estado de ánimo que producen (Mendl \& Paul, 2008; Mendl et al., 2010; Paul et al., 2005).

Cuanto más amplia sea la escala reguladora de los mecanismos homeostáticos en términos conductuales como fisiológicos, mejor estará garantizado el bienestar de los animales. Las condiciones extremas, tales como las bajas temperaturas ambientales, no afectarán al bienestar animal, siempre que sean controlables por medio de algún mecanismo como la construcción de nidos, el metabolismo, el tiriteo, etc. Para saber hasta qué punto el bienestar puede verse afectado por la adaptación a determinadas condiciones, debe conocerse exhaustivamente la especie animal utilizada. Según la especie animal, los factores ambientales tales como el tamaño y estructura de la jaula, luz (intensidad, longitud de onda, fotoperiodo, frecuencia de parpadeo), sonidos, ventilación, etc., tienen idéntica importancia como la presencia o ausencia de sus congéneres, su sexo y la predicción y control del medio ambiente (Morton, 1990).

Es inapropiado el uso del antropomorfismo acrítico en el juicio de la importancia relativa de estos factores, puesto que las condiciones que son adecuadas para conseguir el bienestar humano no tienen por qué serlo también para los animales. Esto es aplicable a la comparación entre las diferentes especies animales y entre las cepas de una misma especie. Hay una serie de investigacio-nes sobre el uso del antropomorfismo en el momento de evaluar los animales en sus distintos aspectos (antroponegación y antropocentrismo animal céntrico, antropocéntrico y heurístico) que deben ser tenidos en cuenta en el momento de analizar la postura epistemo-metodológica de estas investigaciones (De Waal, 1999).

Una forma de no adjudicar al animal predilecciones humanas, es estudiar las preferencias de un animal por ciertas condiciones ambientales, utilizando para ello una prueba de preferencia. Estos ensayos deben utilizarse con cuidado, ya que solo aportan una idea de una elección o preferencia determinada, en un instante dado, por parte del animal. Una ponderación entre el deseo presente del animal y el conocimiento científico es la que indicará cuales preferencias pueden beneficiar al animal a largo plazo (van de Weerd, 1996).

Otra manera de abordar este tema es a través de un sistema de puntuación para medir los signos de estrés o sufrimiento, basado en parámetros comportamentales y clínicos (Kohn et al., 2007). Existen varios indicadores, tales como la piloerección, la reducción de peso corporal, el incremento de la frecuencia cardíaca, la producción de excretas diarreicas, etc. Cada uno de estos sistemas tiene como objetivo estimar el nivel de sufrimiento basándose en parámetros clínicos, fisiológicos y conductuales. Aunque la mayoría de estos parámetros se puede determinar de forma objetiva, la interpretación de los mismos como indicadores del bienestar es subjetiva (Young, 2003).

La toma de indicadores, además de permitir la evaluación de la relación individuo-entorno, en términos de bienestar animal, actúa como diagnóstico de la situación para elaborar intervenciones, de las cuales la más utilizada es el enriquecimiento ambiental (Olfert, 1998).

\section{Enriquecimiento ambiental en animales cautivos}

El enriquecimiento ambiental, según Newberry (1995), se caracteriza como una mejora en el funcionamiento biológico de los animales cautivos, luego de haber introducido modificaciones en su ambiente. La mejora del funcionamiento biológico incluye el éxito de su vida reproductiva y aumento de su eficacia biológica inclusiva y las consecuencias de esto en el mejoramiento de su salud.

En su mayoría, los animales de experimentación son sociables y se benefician de la compañía de sus congéneres o del ser humano. Las experiencias de un animal durante sus fases de desarrollo determinan su comportamiento social. Las condiciones de alojamiento en una instalación de crianza tendrán un impacto sobre su bienestar futuro. Se deben tener en cuenta las mismas consideraciones sobre las necesidades sociales de los animales, tanto como sobre los factores ambientales (iluminación, calefacción, ventilación, habitáculos de contención, etc.). En el caso de animales alojados individualmente, la observación diaria provee una forma alternativa de contacto social para el animal y usualmente facilita las manipulaciones, pues el animal se acostumbra a la presencia del humano (Carlson, 2017; CONADEA, 2016).

Para que los enriquecimientos en términos de intervención sean efectivos, deben realizarse teniendo en cuenta la biología de la especie. En todos los casos, es relevante tener en cuenta, como punto de partida, las características de la cepa ancestral silvestre de la que derivan los animales domésticos. Los ratones silvestres muestran una variación poblacional rítmica, aumentando hasta cierto nivel óptimo, seguido de una declinación rápida, para llegar a un nivel óptimo nuevamente (Baumans et al., 2007).

En la mayoría de los laboratorios, después del destete, los ratones se alojan juntos en grupos de un mismo sexo. Aunque esto no es lo que ocurre con la especie silvestre original, los machos formarán jerarquías, proceso durante el cual puede haber peleas, estrés y heridas. Estas variantes de agresión difieren marcadamente entre las distintas cepas de laboratorio (Nevison, 1999).

Cuando los animales entran en contacto, comienza un período inicial durante el cual establecen sus relaciones sociales (rango de predominio, etc.) y se pueden producir interacciones agresivas. Sin embargo, cuando las condiciones son 
favorables, la organización social se estabiliza y las interacciones estarán basadas más sobre la evasión o la amenaza que sobre el contacto. Si su rutina diaria se desorganiza, si se limitan recursos tales como los alimentos o los espacios de descanso o si los animales están mal agrupados, la jerarquía es perturbada y aumenta la cantidad de interacciones agresivas (van Loo et al, 2001).

El bienestar del animal está amenazado en el aspecto social cuando: a) el espacio es insuficiente para mantener una distancia adecuada para el comportamiento; b) los espacios de alimentación y de descanso para todos los individuos son insuficientes o la alimentación y el descanso no se pueden realizar concurrentemente; c) los reagrupamientos son tan frecuentes que los animales experimentan repetidamente el proceso de estabilización; d) el tamaño de los grupos no es apropiado para las especies. Estos conceptos cuestionan las prácticas intensivas de confinamiento que impiden a los animales ejercer sus actividades sociales y de comportamiento específicas de especie (Ikemoto \& Panksepp, 1992).

Además de su espacio mínimo de descanso, los animales necesitan también lo que se denomina espacio secundario, que les permite libertad de movimiento. Se debe evitar alojar animales individualmente, a menos que sea necesario por razones de salud, de agresión o de investigación. Una excepción importante es el momento del parto, cuando las hembras deben tener sus espacios propios. Los animales alojados solos deben tener algún grado de contacto social con otros de su especie, permitiéndoseles establecer contacto visual. El contacto olfativo y auditivo con otros animales es también deseable (Olfert, et al., 1998).

Beaver (1989) sugiere cinco factores en particular que pueden contribuir al enriquecimiento ambiental: el enriquecimiento del comportamiento, la presencia de congéneres sociales, la existencia de dispositivos físicos, las actividades de búsqueda de alimentos y el control del ambiente. Algunos de estos factores se discuten más adelante.

Baumans (2007) y Berdoy (2003), sostienen que los roedores mantienen una vida parcialmente adaptada a la cautividad, pero aún revelan similitudes con sus contrapartes silvestres. Por esta razón, el medio ambiente del animal de laboratorio debería acomodarse a las necesidades comportamentales y fisiológicas innatas.

Por ejemplo, los roedores son muy susceptibles a los predadores y muestran fuertes respuestas de miedo en situaciones no familiares y cuando no pueden hallar refugio. Muestran tendencia a huir, a morder cuando son manipulados o a quedarse totalmente inmóviles. El animal debería poder sentirse seguro en un ambiente amenazante que pudiera controlar (Poole, 1998).

Según Young (2003), para mejorar el bienestar de los animales y enriquecer el ambiente se emplean diferentes métodos que incluyen desde la mejora en la convivencia social hasta la presencia de elementos estáticos como los juguetes. También se considera como enriquecimiento ambiental el entrenamiento de los animales (Westlund, 2014).

La implementación de un enriquecimiento ambiental específico debe considerar los siguientes principios: 1) mejorar la calidad del cautiverio de manera que el animal tenga una elección mayor de actividades y controles sobre el ambiente social y espacial; 2) aumentar la diversidad comportamental; 3) reducir la frecuencia de comportamientos anormales; 4) incrementar la utilización positiva del ambiente; 5) aumentar la habilidad del animal para que pueda adaptarse a los desafíos (Newberry, 1995; Stauffacher, 1995; Young, 2003).

Según Poole (1998) los animales tienen necesidades fisiológicas y comportamentales. Entre las fisiológicas se incluyen beber, comer, dormir y refugiarse. Entre las comportamentales se incluyen pautas tales como las de comportamiento social, exploración, forrajeo, acicalado, excavación, construcción de nidos, búsqueda de refugio y roedura, fundamentales para el mantenimiento fisiológico normal y psicológico. Estos comportamientos se consideran innatos y se realizan con independencia de sus efectos.

Está reconocido por varios autores, que la interacción social con los congéneres es un factor de bienestar deseable y esencial para los animales de laboratorio. Los ratones alojados juntos deberían ser compañeros de camada; sin embargo, esto no siempre es posible debido a los diseños experimentales y tamaño de las cajas. Lo importante es que el grupo debe ser estable y armonioso (Love 1994; Morton et al., 1993; Stauffacher, 1997a), aunque esto implique disponer de barreras visuales o refugios para ocultarse y evitar las agresiones (Stauffacher 1997b; van de Weerd \& Baumans 1995; van Loo et al., 2002). La presencia de un conespecífico, para un animal social, es el factor de enriquecimiento más estimulante. Mientras que los objetos de enriquecimiento son elementos estáticos y de interés solo para una actividad específica, un compañero social siempre crea nuevas e impredecibles situaciones ante las cuales el animal debe reaccionar. Este tipo de enriquecimiento incrementa los comportamientos de alerta y exploración, los mantiene ocupados y les provee, además, alguna sensación de seguridad (Stauffacher, 2000). Incluso el contacto con humanos (técnicos y cuidadores), en el manejo diario y en el entrenamiento y sociabilización, beneficia al animal y a los resultados de los experimentos debido a que los estimula cognitivamente y permite una interacción más colaborativa con los investigadores (Baumans, 2007; Shepherdson, 1998; van de Weerd \& Baumans, 1995).

De lo expuesto, se deduce la importancia de incorporar como enriquecimiento social el alojamiento de a tríos y ambos sexos en los ratones $\mathrm{y}$, como enriquecimientos físicos, uno del tipo 
refugio y otro del tipo juguete para que puedan refugiarse y roer un objeto. El término juguete (una antropomorfización) es una generalización para muchos objetos. Elementos como cadenas, trapos, bloques de madera o de goma, tubos plásticos, pelotas, etc. son empleados para este tipo de enriquecimiento. La motivación subyacente es el juego. Algunos son de más valor funcional para ciertos animales que para otros y puede haber diferencias en los miembros de un grupo respecto de esta valoración (Held \& Spinka, 2001; Newberry 1995).

El enriquecimiento físico incluye recintos complejos y estímulos sensoriales y nutricionales. La estructuración del ambiente de la caja es más beneficiosa que la provisión de una gran superficie. Salvo para la actividad locomotora, los animales no usan ese espacio, en cambio sí emplean recursos y estructuras internas del área para comportamientos específicos. La mayoría de los roedores dividen su espacio de vivienda en áreas separadas para alimentarse, descansar y excretar. Tales divisiones también permiten a los animales controlar su ambiente, incluso los niveles de iluminación. Estas divisiones pueden ser facilitadas por estructuras internas en la caja: tubos, estantes, cajas y material para nido, plataformas, etc., las que permiten crear áreas de escondite y ocultamiento (Stauffacher, 1997a; Townsend, 1997).

El desarrollo de protocolos para normativizar este tipo de intervenciones está mostrando un fuerte impacto sobre los resultados de las investigaciones (Slater \& Cao, 2015).

\section{Discusión y conclusiones}

Hasta aquí, solo hemos introducido las modificaciones que este enfoque produce en nuestra visión del animal utilizado en investigación. Cuando a partir de esta visión se rediseñan las experiencias, ¿̇e produce un cambio?, ¿̇o solo es lo mismo de siempre pero con un lenguaje más filosófico y políticamente correcto?

Estas no son solo preguntas de relevancia teórica. Si diseñar las investigaciones teniendo en cuenta el bienestar animal produce distintos resultados, entonces todos los resultados obtenidos a partir del reactivo biológico pierden generalidad, es decir solo serán válidos para las condiciones de bienestar en que se obtuvieron. Si estas condiciones de bienestar son tales que aplican a cualquier conjunto de variables ambientales, nada cambia, aunque, a priori, no se puede presuponer que lo sean.

No importa qué tan probable sea esta discrepancia entre las investigaciones que tuvieron en cuenta el bienestar animal y las que no. El impacto del cambio de punto de vista es sumamente relevante, al menos de dos formas. Por un lado, nos lleva a formular las hipótesis no para el animal-reactivo, uniforme, controlado por las condi- ciones del ambiente, sino para uno proactivo, con conductas intrínsecamente motivadas con una dinámica histórica y con sintiencia. Por otro lado, deberíamos realizar investigaciones específicas (o agregar objetivos a las no específicas) sobre cómo influye en los resultados este cambio de paradigma.

Por ejemplo, cuando se comparan los comportamientos de cepas de ratones de experimentación endocriados y exocriados, sometidos a un ensayo de campo abierto en el que se pueden evaluar diferentes conductas, y que han sido criados en un ambiente con distintos tipos de enriquecimiento ambiental, versus una crianza estándar (sin enriquecimiento), se observa que la variación de las conductas de una y otra cepa para los distintos comportamientos, es menor en los grupos criados con enriquecimiento que en los que no lo tienen. Esto nos permite sugerir que el enriquecimiento no suma una variable más que pudiera alterar los resultados de un ensayo. Además, las respuestas son diferentes según el tipo de enriquecimiento realizado. En los grupos con enriquecimiento del ambiente se evidencia menor frecuencia de manifestación de algunas conductas, comparado con los grupos control, resultando en una mejor adaptación a la nueva situación (Maschi, 2017).

Los ratones criados con distintos enriquecimientos actúan produciendo pautas de comportamiento diferentes, dependiendo si pertenecen a una cepa endocriada o a una línea exogámica; en algunos, provocan que se manifiesten, con mayor frecuencia, algunas conductas mientras que, en otros, disminuyen (Lang \& Vessell, 1976; Maschi, 2017).

Por lo tanto, antes de implementar un programa de enriquecimiento ambiental, deben evaluarse las diferentes posibilidades de modificación del microambiente para cada cepa o stock en particular, dado que, si bien el enriquecimiento genera conductas y beneficia el bienestar de los animales, los comportamientos resultantes son diferentes para cada modelo de ratón.

Surge entonces la necesidad de elaborar indicadores específicos del bienestar para animales usados en investigación, que permitan por un lado identificar y remediar las condiciones de pobre bienestar, y por otro, comunicar con precisión las condiciones de los grupos a partir de los cuales se hacen las investigaciones.

Los animales que se proveen a los investigadores deberán no solo ser acompañados con certificaciones sobre la cepa y condiciones de crianza, sino también sobre el tipo y modalidad de enriquecimiento y los indicadores de bienestar animal al momento de iniciar su utilización.

Se puede aseverar que está cambiando la definición de animal: ya no solo es una anatomía y una fisiología sino también, y tal vez con mayor importancia, un comportamiento y una sintiencia. Esto equivale a decir que, en el futuro, el bienestar 
animal no solo debe estar en la nota ética, sino que debe ser definitivamente parte inexcusable de la sección metodológica de los estudios experimentales.

\section{Agradecimientos}

A todo el personal del área de producción del Laboratorio de Animales de Experimentación (LAE). Este trabajo forma parte de la tesis de doctorado de Fabricio A. Maschi (Facultad de Ciencias Veterinarias, Universidad Nacional de La Plata, 2017) y parcialmente subsidiado con recursos propios del LAE.

\section{Conflicto de intereses}

Todos los autores declaran que no existe conflicto de intereses, incluyendo entre estos últimos las relaciones financieras, personales o de otro tipo con otras personas $\mathrm{u}$ organizaciones que pudieran influir de manera inapropiada en el trabajo.

\section{Bibliografía}

ASAB (Association for the Study of Animal Behaviour). 1986. Guidelines for the use of animals in research. Animal Behaviour. 34(1):31518. doi: 10.1016/0003-3472(86)90056-4

Baumans V, Coke C, Green J, Moreau E, Morton D, Patterson-Kane M, Reinhardt A, Reinhardt V, Van Loo P. 2007. Making -lives easier for animals in research labs. Discussions by the Laboratory Animal Refinement \& Enrichment Forum. Washington DC, Animal Welfare Institute (AWI). pp. 105. Disponible en

https://www.parsemus.org/wp-content/uploads/ 2012/11/Making_Lives_Easier1.pdf

[Accedido 22/05/2019]

Baumans V. The welfare of laboratory mice. En: Kaliste E. 2007. The welfare of laboratory animals. Dordrecht, Springer, pp. 119-52. Disponible en: https://link.springer.com/content/pdf/

10.1007\%2F978-1-4020-2271-5.pdf

[Accedido 22/05/2019]

Beaver BV. 1989. Environmental enrichment for laboratory animals. Institute Laboratory Animal Resources (ILAR) News. 31 (2):5-11.

doi: 10.1093/ilar.31.2.5

Berdoy M. 2003. Ratlife. [En línea]. Disponible en: http://www.ratlife.org [Accedido 29/09/2018]

Brambell Rogers FW. 1965. Report of the technical committee to inquire into the welfare of animal slept under intensive livestock husbandry systems. London, Her Majesty's Stationery Office.
Broom D. 1986. Indicators of poor welfare. British Veterinary Journal. 142(6):524-26.

doi: 10.1016/0007-1935(86)90109-0

Broom D. 2016. Sustentabilidad y sintiencia en relación con el bienestar animal. Conferencia con motivo de recibir el Doctorado Honoris causa, Universidad de Buenos Aires (UBA). Revista InfoVet. Número especial, Facultad de Ciencias Veterinarias, Universidad de Buenos Aires.

Carlson BA. 2017. Early life experiences have complex and long-lasting effects on behavior. Proceeding of the National Academy of Sciences of the Unites States of America (PNAS). 114(44): 11571-3. doi: 10.1073/pnas.1716037114

Cardoso CVP, Almeida AECC. 2014. Laboratory animal: biological reagent or living being? Brazilian Journal of Medical and Biological Research. 47(1):19-23. doi: 10.1590/1414-431X20133365

Chamove AS. 1989. Environmental enrichment: A review. Animal Technology, 40(3):155-78. Reproduced with permission of the Institute of Animal Technology in Animal Welfare Institute. Disponible en: https://awionline.org/content/environmentalenrichment-review [Accedido 22/05/2019]

Chandrasekera Ch. 2016. From sentience to science: Limits of anthropocentric cognition. Animal Sentience. 5(2). Disponible en: https://animalstudiesrepository.org/animsent/ vol1/iss5/2/ [Accedido 22/05/2019]

CONADEA (Comisión nacional de experimentación animal). 2016. Ley de protección para los animales de experimentación utilizados con fines científicos y educativos (Proyecto 6758-D-2016) [En línea] Disponible en:

https://www.diputados.gov.ar/proyectos/

proyecto.jsp? $\exp =6758-\mathrm{D}-2016$

[Accedido 12/11/2018].

Dawkins MS. 1990. From an animal's point of view: motivation, fitness and animal welfare. Behavioral and Brain Sciences. 13(1):1-9.

doi: 10.1017/So140525Xo0077104

De Grazia D. 1996. Taking animals seriously: Mental life and moral status. New York, Cambridge University Press. Disponible en:

http://hdl.handle.net/10822/751779

[Accedido 12/11/2018].

De Mello M. 2012. Animals and society: an introduction to human-animal studies. New York, Colombia University Press.

De Waal FBM. 1999. Anthropomorphism and anthropodenial: consistency in our thinking about humans and other animals. Philosophical Topics. 27 (1):255-80. 
Duncan IJH. 1998. Behaviour and behaviour needs. Poultry Science. 77(12):1766-72.

doi: $10.1093 / \mathrm{ps} / 77.12 .1766$

Fraser D. 2004. Applying science to animal welfare standards. Global conference on animal welfare: an OIE initiative. Paris, pp. 121-32.

Hau J, Shapiro SJ. The welfare of non human primates. En: Kaliste E. 2007.The welfare of laboratory animals. Dordrecht, Springer, pp. 291314. Disponible en:

https://link.springer.com/chapter/

10.1007\%2F978-1-4020-2271-5_13

[Accedido 12/11/2018].

Hawkins P. 2002. Recognizing and assessing pain, suffering and distress in laboratory animals: a survey of current practice in the UK, with recommendations. Laboratory Animals. 36(4): 378-95. doi: 10.1258/002367702320389044

Hawkins P, Morton DB, Burman O, Dennison N, Honess $\mathrm{P}$, Jennings $\mathrm{M}$, Lane S, Middleton V, Roughan JV, Wells S, Westwood K. 2011. A guide to defining and implementing protocols for the welfare assessment of laboratory animals: 11th report of the BVAAWF/FRAME/RSPCA/UFAW Joint Working Group on Refinement Laboratory Animals. 45(1):1-13. doi: 10.1258/la.2010.010031

Held SDE, Spinka M. 2011. Animal play and animal welfare. Animal Behaviour. 81(5):891-9. doi: 10.1016/j.anbehav.2011.01.007

Hughes BO. 1976. Behaviour as an indicator of welfare. Proceeding of the fifth european poultry confererence. Malta, pp. 1005-18.

Ikemoto S, Panksepp J. 1992. The effects of early social isolation on the motivation for social play in juvenile rats. Developmental Psychobiology. 25(4): 261-74. doi: 10.1002/dev.420250404

ISAE (International Society for Applied Ethology). 2017. Guidelines for ethical treatment of animals in applied animal behaviour and welfare research. [En línea] Disponible en:

https://www.applied-ethology.org/

Ethical_Guidelines.html [Accedido 24/10/2018]

Kohn DF, Martin ME, Foley PL, Morris TH, Swindle MM, Voglet GA, Wicson SK. 2007. Guidelines for the assessment and management of pain in rodents and rabbits. Journal of the American Association for Laboratory Animals Science 46-(2):97-108.

Lang CM, Vessell ES. 1976. Environmental and genetics factors affecting laboratory animals: impact on biomedical research. Introduction. Federal Proceedings. 35(5):1123- 4.
Langford DJ, Bailey AL, Chanda ML, Clarke SE, Drummond TE, Echols S, Glick S, Ingrao J, Klassen-Ross T, La Croix-Fralish ML, Matsumiya L, Sorge RG, Sotocinal SG, Tabaka JM, Wong D, van den Maagdenberg AM, Ferrari MD, Craig KD, Mogil JS. 2010. Coding of facial expressions of pain in the laboratory mouse. Nature Methods 7 (6):447-9. doi: 10.1038/nmeth.1455

Love JA. 1994. Group housing: Meeting the physical and social needs of the laboratory rabbit. Laboratory Animal Science. 44(1):5-11.

Maschi F. 2017. El efecto del enriquecimiento ambiental sobre la variabilidad de parámetros fisiológicos y conductuales en ratones de laboratorio. Tesis de doctorado. Facultad de Ciencias Veterinarias. Universidad Nacional de La Plata.

http://sedici.unlp.edu.ar/handle/10915/63094

[Accedido 22/05/2019].

Mason, P. 1997. The brown dog affair: the story of a monument that divided the nation. Londres, Two Sevens Publishing.Mellor DJ, Reid CSW. Concepts of animal well-being and predicting the impact of procedures on experimental animals. En: Baker RM, Jenkin G, Mellor DJ. 1994. Proceedings of the improving the well-being of animals in the research environment. Glen Osmond, Australia, Australian \& New Zealand Council for the Care of Animals in Research and Teaching (ANZCCART), pp.3-18.

Mellor DJ. 2016. Updating animal welfare thinking: moving beyond the "five freedoms" towards "a life worth living". Animals 6(3):21. doi: 10.3390/ani6030021

Mendl M, Paul ES. 2008. Do animals live in the present?: Current evidence and implications for welfare. Applied Animal Behaviour Science. 113(4): 357-82. doi: 10.1016/j.applanim.2008.01.013

Mendl M, Burman OHP, Parker RMA, Paul ES. 2009. Cognitive bias as an indicator of animal emotion and welfare: emerging evidence and underlying mechanisms. Applied Animal Behaviour Science. 118(3-4):161-81.

doi: 10.1016/j.applanim.2009.02.023

Mendl M, Burman OHP, Paul ES. 2010. An integrative and functional frame work for the study of animal emotion and mood. Proceeding of the Royal Society B. doi: 10.1098/rspb.2010.0303

Morton DB. 1990. Adverse effects in animals and their relevance to refining scientific procedures. Alternatives to Laboratory Animals (ATLA). 18:2939.

Morton DB, Jennings M, Batchelor GR, Bell D, Birke L, Davies K, Eveleigh JR, Gunn D, Heath M, 
Howard B, Koder P, Phillips J, Poole T, Sainsbury AW, Sales GD, Smith DJA, Stauffacher M, Turner RJ. 1993. Refinements in rabbit husbandry. Second report of the BVAAWF/FRAME/RSPCA/ UFAW Joint Working Group on Refinement. Laboratory Animals. 27 (4):301-29

Nevison CM, Hurst JL, Barnard CJ. 1999. Why do male ICR(CD-1) mice perform bar-related (stereotypic) behaviour?. Behavioural Processes. 47-(2):95-111.

doi: 10.1016/So376-6357(99)ooo53-4

Newberry RC. 1995. Environmental enrichment: increasing the biological relevance of captive environments. Applied Animal Behaviour Science. 44(2-4):229-43.

doi: 10.1016/0168-1591(95)oo616-Z

Olfert ED, Cross BM, Mc William AA. 1998. Manual sobre el cuidado y uso de los animales de experimentación (Estol L, Dugas R, Traducción en español), Ontario, Canadian Council on Animal Care (CCAC). Disponible en:

http://www.fcv.unl.edu.ar/comite/

Manualsobreelcuidadoyusodeanimalesdeexperime ntacionConsejo\%20Canadiense.pdf

[Accedido 22/05/2019].

OMS (Organización Mundial de la Salud). [En línea]. Disponible en: http://www.who.int [Accedido 29/9/2018].

Paul ES, Harding EJ, Mendl M. 2005. Measuring emotional processes in animals: the utility of a cognitive approach. Neuroscience \& Biobehavioral Reviews. 29(3):469-91.

doi: 10.1016/j.neubiorev.2005.01.002

Poole T. 1998. Meeting a mammal's psychological needs: basic principles. En: Second nature: environmental enrichment for captive animals. Eds. Sheperdson D. Mellen J, Hutchins M. Washington, Smithsonian Institution Press. pp. 83-96.

Proctor H. 2012. Animal sentience: where are we and where are we heading? Animals. 2(4):628-39. doi: $10.3390 /$ ani2040628

Segerdahl P. 2007. Can natural behavior be cultivated? The farm as local human/animal culture. Journal of Agricultural and Environmental Ethics. 20(2):167-93.

Shepherdson DJ. Tracing the path of environmental enrichment in zoos. En: Shepherdson DJ, Mellen JD, Hutchins M. 1998. Second nature: environmental enrichment for captive animals. Washington DC, Smithsonian Institution Press, pp. 1-12.
Singer, P. 2015. Animal liberation: The definitive classic of the animal movement. New York, Open Road Media.

Slater AM, Cao L, 2015. A protocol for housing mice in an enriched environment. Journal of Visualized Experiments. 100:e52874.

doi: $10.3791 / 52874$

Sorensen D. Animal welfare: an introduction. En: Kaliste E. 2007.The welfare of laboratory animals. Dordrecht, Kluwer Academic Publisher, pp. 3-14.

Stauffacher M. 1995. Environmental enrichment, fact and fiction. En: Environmental enrichment information resources for laboratory animals. Animal Welfare Information Center (AWIC) Resource Series. 2:145-9.

Stauffacher M. Housing requirements: What ethology can tell us. En: van Zutphen LFM, Balls $\mathrm{M}$, eds. Animal alternatives, welfare and ethics. 1997a. Amsterdam, Elsevier Science BV. pp. 17986.

Stauffacher M. Comparative studies on housing conditions. En: O'Donoghue PN, ed. 1997b Harmonization of laboratory animal husbandry. London, Royal Society of Medicine Press, pp. 5-9.

Stauffacher M. Refinement in rabbit housing and husbandry. En: Balls M, van Zeller AM, Halder M, eds. 2000. Progress in the reduction, refinement and replacement of animal experimentation, developments in animal and veterinary sciences. Amsterdam, Elsevier Science BV. pp. 1269-77.

Townsend P. 1997. Use of in-cage shelters by laboratory rats. Animal Welfare. 6(2):95-103.

Turner J. 1999. Factory farming and the environment: a report for compassion in world farming trust. Petersfield, Hampshire, Compassion in World Farming Trust.

van de Weerd HA, Baumanns V. Environmental enrichment in rodents. En: Environmental enrichment resources for laboratory animals. 1995. Animal Welfare Information Center (AWIC) Resource Series. 6(2):95-103.

van Loo PLP, Kruitwagen CLJJ, Koolhaas JM, van de Weerd HA, van Zutphen LFM, Baumans V. 2002. Influence of cage enrichment on aggressive behaviour and physiological parameters in male mice. Applied Animal Behaviour Science. 76(1):6581. doi: 10.1016/So168-1591(01)00200-3

van Loo PLP, Mol JA, Kolhaas JM, van Zutphenbf, Baumans V. 2001. Modulation of aggression in male mice: influence of group size and cage size. Physiology and Behaviour. 72(5):675-83. 
Westlund K. 2014. Training is enrichment and beyond. Applied Animal Behaviour Science. 152:16. doi: 10.1016/j.applanim.2013.12.009

World Veterinary Association. 1989. World Veterinary Association policy statement on animal welfare, well-being, and ethology. Institute for Laboratory Animal Research (ILAR) Journal. 31(4): 29-30. doi: 10.1093/ilar.31.4.29
Young RJ. Environmental enrichment for captive animals. 2003. Universities Federation for Animal Welfare (UFAW). Ames, Iowa, Blackwell Publishing Professional. 